\title{
Cryptococcus Pneumonia Complicating Churg-Strauss Syndrome
}

\author{
Mayar Al Mohajer ${ }^{\mathrm{a}, \mathrm{b}}$, Richard J. Hamill ${ }^{\mathrm{a}}$, Rabih O. Darouiche
}

\begin{abstract}
Crytpococcus neoformans usually infects individuals who are HIVinfected or immunosuppressed for other reasons and clinically presents as meningoencephalitis, pneumonia or disseminated disease. We describe a 57-year-old man with history of asthma, allergic rhinosinusitis, nasal polyps and chronic receipt of low dose steroids who presented to the emergency department with fever, dry cough, respiratory distress and hematuria. His laboratory studies showed leukocytosis and eosinophilia with a negative HIV antibody test. CT scan of the chest showed right lower lobe patchy opacities. The patient deteriorated during hospitalization, developed hemoptysis and hypoxia, and was electively intubated. Repeat CT scan of the chest showed diffuse alveolar and interstitial opacities with mediastinal lymphadenopathy. The cytoplasmic anti-neutrophil cytoplasmic antibody (C-ANCA) was markedly elevated and the patient was diagnosed with Churg-Strauss Syndrome (CSS). Culture of bronchoalveolar lavage fluid yielded growth of Cryptococcus neoformans. The patient responded well to treatment with cyclophosphamide, corticosteroids, plasmapharesis and fluconazole. This case highlights the possibility that Cryptococcus neoformans can either infect or colonize patients who are immunosuppressed, including those with CSS.
\end{abstract}

Keywords: Cryptococcus neoformans; Churg-Strauss Syndrome; Vasculitis

\section{Introduction}

Crytpococcus neoformans can cause pneumonia in patients with congenital or acquired defects in their host defenses.

\footnotetext{
Manuscript accepted for publication October 25, 2012

${ }^{a}$ Infectious Disease Section, Michael E. DeBakey Veterans Affairs Medical Center and Baylor College of Medicine, Houston, Texas, USA

${ }^{\mathrm{b}}$ Corresponding author: Mayar Al Mohajer, 1 Baylor Plaza, Department of Infectious Diseases Houston, TX 77030. USA.

Email: mohajer@bcm.edu
}

doi: http://dx.doi.org/10.4021/jmc755w
We present a case of pulmonary cryptococcosis and ChurgStrauss syndrome that occurred following prolonged corticosteroid use and was successfully treated.

\section{Case Report}

A 57-year-old African American male, prior smoker, with a history of chronic obstructive pulmonary disease, asthma, nasal polyps and allergic rhinosinusitis, was admitted to our tertiary care hospital for fever and respiratory distress. He had had 1 week of fever, dry cough and shortness of breath, as well as periumbilical abdominal pain, nausea, vomiting and hematuria. He was on chronic low dose prednisone (10 mg daily) and montelukast as well as inhalers. On examination, he was found to be febrile $\left(39.6^{\circ} \mathrm{C}\right)$ with a pulse rate of $101 / \mathrm{min}$ and a blood pressure of $142 / 86 \mathrm{mmHg}$. The patient had diffuse crackles and wheezing. Abdominal examination revealed mild periumbilical tenderness. His labs showed elevated white blood count of 14,400 cells/mL, mild anemia (hemoglobin, $11.9 \mathrm{~g} / \mathrm{dL}$ ) and eosinophilia of 13\%. Electrolytes, renal function and liver enzymes were normal. Urinalysis showed 1,192 red blood cells and 49 white blood cells per high power field with occasional bacteria.

Chest radiograph showed mild upper lobe emphysematous changes. Chest CT scan showed patchy airspace opacities in the right lower lobe. Abdominal and pelvic CT scans were normal. Sinus CT scan showed near complete opacification of all sinuses. The patient was started on broad spectrum antibiotics for concern of pneumonia and urinary tract infection. On day 4 of admission, he developed frank hemoptysis with hypoxia, requiring $50 \% \mathrm{O} 2$ by Venturi mask. He was electively intubated and bronchoscopy was performed which showed diffuse alveolar hemorrhage. No biopsy was done given his anemia and hemoptysis. Repeat chest CT scan (Fig. 1) showed interval progression of diffuse alveolar and interstitial opacities with mediastinal lymphadenopathy. Tests for HIV and strongyloides were negative. Blood, urine and sputum cultures were negative. The anti-nuclear antibody profile, rheumatoid factor, anti-glomerular basement membrane antibody, perinuclear anti-neutrophil cytoplasmic antibodies (P-ANCA), anti-proteinase-3 (PR3) and complements 


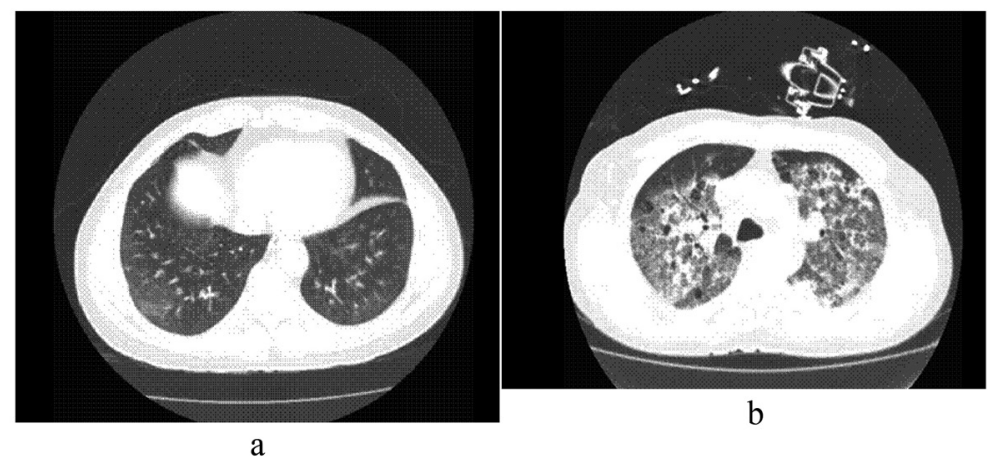

Figure 1. (a) Chest CT on admission, (b) Chest CT on day 6.

were normal. The cytoplasmic anti-neutrophil cytoplasmic antibodies (C-ANCA) and the myeloperoxidase antibodies (MPO) were markedly elevated. Bronchoalveolar lavage fluid culture was positive for Cryptococcus neoformans. Serum Cryptococcal polysaccharide titer was negative.

A clinical diagnosis of Churg-Strauss syndrome (CSS) and pulmonary cryptococcosis was made. The patient was started on high dose intravenous corticosteroids, cyclophosphamide, plasmapharesis and fluconazole. He was intubated three times during his admission and underwent cardiopulmonary resuscitation for cardiac arrest. He improved clinically and was discharged after 4 weeks on prednisone $60 \mathrm{mg}$ daily, fluconazole $400 \mathrm{mg}$ daily and dapsone (100 mg daily) for pneumocystis jirovecii pneumonia prophylaxis.

\section{Discussion}

Cryptococcus neoformans is an uncommon cause of pneumonia in HIV- negative patients. Cryptococcal pneumonia has been associated with many immunosuppressive conditions, including lymphoproliferative disorders, sarcoidosis, corticosteroid therapy, Hyper-IgM syndrome, Hyper-IgE syndrome, monoclonal antibody use, systemic lupus erythematosus, idiopathic CD4+ T-cell lymphocytopenia, diabetes mellitus, organ transplantation, peritoneal dialysis and cirrhosis $[1,2]$.

To our knowledge, only one other case in the medical literature had reported pulmonary cryptococcosis complicating Churg Strauss syndrome [3]. Churg-Strauss syndrome is a multisystem disorder characterized by chronic rhinosinusitis, asthma, and prominent peripheral blood eosinophilia. Churg-Strauss syndrome can develop in people with asthma who are steroid-dependent and who are treated with montelukast upon reduction in their steroid use.

Pulmonary cryptococcosis usually presents with cough and dyspnea. Fever, night sweats, weight loss and pleuritic pain are uncommon manifestations [2]. Serum cryptococcal antigen is positive in only half of the cases of pulmonary cryptococcosis while it is almost always positive in patients with disseminated disease (52\% vs. $92 \%$ ). Patients with mild to moderate pulmonary disease are usually treated with fluconazole (400 mg daily) for 6 - 12 months while patients with severe disease are treated like central nervous system disease with amphotericin B plus flucytosine followed by fluconazole [4].

The significance of the isolation of Cryptococcus neoformans in our patient is not clear. While it could represent a significant infection in our immunocompromised patient given the history of prolonged steroid use and recent diagnosis of Churg Strauss syndrome, it can also be a colonizing pathogen found incidentally in bronchoscopic specimens. In one study in 1977, 43 patients had Cryptococcus neoformans isolated from the respiratory tract [5]. Of those, 13 patients had no evidence of parenchymal disease and were not treated with antifungal medications (fluconazole was not available at that time). None of the patients developed symptoms suggesting disseminated disease or meningitis after follow up 13 - 57 months. Our patient was treated, given his immunosuppressive status and the availability of a safe well-tolerated drug (fluconazole).

In conclusion, cryptococcal pneumonia can be seen in immunocompromised patients including those with ChurgStrauss syndrome. Careful diagnosis and prompt treatment is crucial.

\section{Disclosure}

The authors report no conflict of interest and have not received any funding for this study.

\section{References}

1. Perfect JR. Cryptococcus neoformans. 7th ed. In: Mandell GL, Bennett JE, Dolin R, editors. Principles and practice of infectious diseases, Philadelphia: Churchill Livingstone; 2010; 3287-3203.

2. Duperval R, Hermans PE, Brewer NS, Roberts GD. 
Cryptococcosis, with emphasis on the significance of isolation of Cryptococcus neoformans from the respiratory tract. Chest. 1977;72(1):13-19.

3. Baddley JW, Perfect JR, Oster RA, Larsen RA, Pankey GA, Henderson H, Haas DW, et al. Pulmonary cryptococcosis in patients without HIV infection: factors associated with disseminated disease. Eur J Clin Microbiol Infect Dis. 2008;27(10):937-943.
4. Perfect JR, Dismukes WE, Dromer F, Goldman DL, Graybill JR, Hamill RJ, Harrison TS, et al. Clinical practice guidelines for the management of cryptococcal disease: 2010 update by the infectious diseases society of america. Clin Infect Dis. 2010;50(3):291-322.

5. Amundson DE. Cavitary pulmonary cryptococcosis complicating Churg-Strauss vasculitis. South Med J. 1992;85(7):700-702. 